\title{
Composição florística das Plantas daninhas na Cultura de Guaraná (Paullinia cupana), No Estado do Amazonas ${ }^{1}$
}

\author{
Floristic Composition of Weeds in Guarana (Paullinia cupana) Crop in \\ Amazonas, Brazil
}

ALBERTINO, S.M.F. ${ }^{2}$, SILVA, J.F. ${ }^{3}$, PARENTE, R.C. ${ }^{4}$ e SOUZA, L.A.S. ${ }^{5}$

\begin{abstract}
RESUMO - O estudo da composição da flora de plantas daninhas de uma área ou de uma região agrícola é importante, por identificar a diversidade de espécies e contribuir para recomendação de estratégias de manejo a serem empregadas nos sistemas agrícolas. Este trabalho teve o objetivo de identificar a composição florística de plantas daninhas em plantios de guaraná em cinco municípios (Coari, Iranduba, Maués, Presidente Figueiredo e Urucará) do Estado do Amazonas. As plantas daninhas foram amostradas em áreas de produtores desses municípios e identificadas por classe, família e espécie. Foram coletados 14.707 indivíduos, distribuídos em 40 famílias e 87 espécies, das quais 70 eram Dicotiledôneas, 13 Monocotiledôneas e quatro Pteridófitas. As Dicotiledôneas apresentaram maior número de família e as Monocotiledôneas de indivíduos. As Pteridófitas ocorreram de forma inexpressiva em todos os municípios. As famílias Poaceae e Asteraceae apresentaram maior número de espécies. Panicum pilosum foi a espécie com maior número de indivíduos, freqüência, densidade e índice de valor de importância. Panicum laxum sobressaiu em Urucará e Iranduba; Scleria malaleuca, em Coari; e Chamaesyce hirta, em Maués. Homolepis aturensis, Paspalum conjugatum e Spermacoce capitata ocorreram nos cinco municípios. Em Maués foi encontrada a maior diversidade de espécies, e em Coari, o maior número de indivíduos. O maior índice de similaridade foi entre Iranduba e Presidente Figueiredo (45\%), e o menor, entre Iranduba e Coari $(21,43 \%)$.
\end{abstract}

Palavras-chave: guaranazeiro, fitossociologia, invasoras, freqüência, Amazônia.

\begin{abstract}
The study of the floristic composition of weeds of an area or agricultural region is important since it identifies the diversity of species and provides management strategies to be used under various agricultural system conditions. The objective of this work was to identify the floristic composition of weeds occurring in guaraná crops in five counties (Coari, Iranduba, Maués, Presidente Figueiredo and Urucará) located in the state of Amazonas, Brazil. Samples of weeds were taken from guarana growing areas in the five counties and identified by class, family and species. A total of 14,707 individual plants were collected and grouped into 40 families and 87 species, as follows: 70 Dicotyledonous, 13 Monocotyledonous and 4 Pteridophyts. The Dicotyledonous class presented the largest number of families and the monocotyledonous the largest number of individual plants, except in Maues and Iranduba. The number of Pteridophyts was inexpressive in all the counties. The Poaceae and Asteraceae families presented the largest number of species. Poaceae was found in all the counties, presenting the largest number of species in Maues. Panicum pilosum was the species with the largest number of individual plants, frequency, density and value of importance index, while Panicum laxum presented an expressive number of species in Urucará and Iranduba. Scleria malaleuca presented an expressive number in Coari and Chamaesyce hirta in Maués. Homolepis aturensis, Paspalum conjugatum and Spermacoce capitata were found in the five counties. The largest species diversity was found in Maues whereas the largest number of individual plants was found in Coari. The largest similarity index was found between Iranduba and Presidente Figueiredo (45\%) and the smallest between Iranduba and Coari (21.43\%).
\end{abstract}

Key words: guarana crop, phyto-sociology,weeds,frequency, Amazonian.

1 Recebido para publicação em 11.2.2004 e na forma revisada em 10.9.2004.

Parte da dissertação do primeiro autor em Ciências Agrárias.

2 Eng.-Agr., M.S., <jfsilva@ufam.edu.br>; ${ }^{3}$ Eng.-Agr., D.S., Universidade Federal do Amazonas - FCA-UFAM, Av. Gal. Rodrigo J. Ramos 3000, 69077-000 Manaus-AM, <propesp@ufam.edu.br>; ${ }^{4}$ Estatística, D.S., Universidade Federal do Amazonas - UFAM-ICE; ${ }^{5}$ Eng.-Agr., M.S., Professora Substituta da Universidade Federal do Amazonas - UFAM-FCA. 


\section{INTRODUÇÃO}

A caracterização de comunidades de plantas daninhas de uma área ou de uma região agrícola é importante, por contribuir na detecção de problemas e na escolha de estratégias de manejo e de controle a serem empregadas nos sistemas agricolas (Yanagizawa \& Maimoni-Rodella, 1999).

Levantamentos de plantas daninhas foram feitos em lavouras de soja (Pereira et al., 2000), cana-de-açúcar (Campelo, 1988), feijão (Brandão et al., 1986), mandioca (Alcantara \& Carvalho, 1983), árvores frutíferas (Yanagizawa \& Maimoni-Rodella, 1999) e pastagens (Modesto Junior \& Mascarenhas, 2001). Também, fez-se levantamento de ocorrência de plantas daninhas em áreas agrícolas do Paraná (Dombroswski, 1989), Minas Gerais (Brandão et al., 1986) e São Paulo (Leitão et al., 1972). Entretanto, no Estado do Amazonas, estudos nessa linha foram encontrados para as culturas do cupuaçu (Souza, 2000), em área de sistemas agroflorestais de cupuaçuzeiro e pupunheira (Souza et al., 2003), porém nenhum trabalho para a cultura do guaraná.

É importante a realização e atualização desses levantamentos, devido à dinâmica da flora infestante da lavoura, que na região do trópico úmido, como a Amazônia, apresenta trocas em menor espaço de tempo.

Este trabalho teve o objetivo de identificar a composição florística de plantas daninhas que ocorrem nos plantios de guaraná em cinco municípios do Estado do Amazonas.

\section{MATERIAL E MÉTODOS}

As coletas das amostras de plantas daninhas e suas coordenadas geoprocessadas foram feitas nos municipios de Coari (S 46’57”, W 634'30"), Iranduba (S 38'48”, W

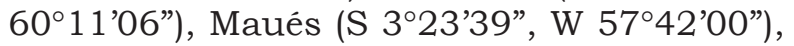
Presidente Figueiredo (S 1 $1^{\circ} 57^{\prime} 51^{\prime \prime}$, W $\left.60^{\circ} 13^{\prime} 49^{\prime \prime}\right)$ e Urucará (S 2³0’41”, W 5742’43”), no Estado do Amazonas.

As áreas plantadas com guaranazeiros foram preparadas a partir da derrubada da floresta primária há mais de dez anos. As idades das plantas de guaraná são superiores a oito anos, exceto as do município de Presidente
Figueiredo, que têm idade de quatro anos e foram plantadas espaçadas 5 × $5 \mathrm{~m}$ entre si. $\mathrm{O}$ manejo das plantas daninhas nos municípios de Coari, Iranduba, Urucará e parte de Maués é feito com terçado (facão) uma ou duas vezes ao ano, na projeção da planta de guaraná. Nos municípios de Presidente Figueiredo e na outra parte de Maués, o manejo das plantas é feito com uso de herbicida e também adubação química e orgânica. Nos demais municípios não se usa adubo e raramente podam-se os ramos das plantas de guaraná.

Em cada local de coleta foi arremessado um quadrado de madeira de $0,60 \times 0,60 \mathrm{~m}$, em duas linhas transversais em formato de " $x$ ", por 20 vezes, atirado ao acaso, perfazendo um total de 20 parcelas e área amostral de $7,2 \mathrm{~m}^{2}$. As plantas dentro do quadrado foram cortadas rente ao solo, contadas e separadas, sendo preparadas exsicatas de cada espécie. A identificação das plantas foi feita pela classe, família e espécie, por comparação com o auxílio da literatura, ou elas foram enviadas à especialista. As plantas não identificadas foram encaminhadas para centros especializados, que trabalham com as familias ainda não identificadas. As exsicatas das plantas identificadas e numeradas foram depositadas no herbário da UFAM.

A avaliação das comunidades de plantas daninhas foi feita utilizando-se os seguintes parâmetros: freqüência, densidade, abundância, freqüência relativa, densidade relativa, abundância relativa e índice de valor de importância. Esses parâmetros foram estimados de acordo com Brandão et al., (1998), e também foi calculado o índice de similaridade entre os municípios, segundo a fórmula proposta por Sorensen (1972).

$\mathrm{Na}$ comparação entre municípios, dentro de cada espécie, utilizou-se o teste estatístico não-paramétrico de Kruskall-Wallis, fazendo-se comparações múltiplas com nível de significância de $5 \%$.

\section{RESULTADOS E DISCUSSÃO}

Nos cinco municípios avaliados, foram coletados 14.707 indivíduos distribuídos em 40 famílias, representadas por 87 espécies, sendo 70 Dicotiledôneas (80,5\%), 13 Monocotiledôneas (15\%) e 4 Pteridófitas (4,5\%). 
Dicotiledônea também foi a classe que apresentou o maior número de famílias nos cinco municípios estudados (Tabela 1). Esses resultados estão de acordo com os obtidos por Souza et al. (2003) em agrossistemas de cupuaçuzeiro e pupunheira no Amazonas, que encontraram maior número de espécies pertencente à classe das Dicotiledôneas. Modesto Junior \& Mascarenhas (2001), em levantamentos de plantas daninhas em pastagens no nordeste paraense, também relataram resultados semelhantes aos encontrados neste trabalho: das 36 espécies de plantas daninhas, 7 eram monocotiledôneas (20\%) e 29 dicotiledôneas (80\%). Yanagizava \& Maimoni-Rodella (1999) identificaram 54 espécies - 44 eram dicotiledôneas (81\%) e 10 monocotiledôneas $(19 \%)$ - em cultivo de árvores frutiferas em Botucatu-SP.

As monocotiledôneas apresentaram menor número de espécies, porém obtiveram maior número de indivíduos (8.395), com 57\% do total das três classes juntas (Figura 1).

Nas famílias Poaceae e Asteraceae foi registrado o maior número de espécies, ambas

Tabela 1 - Número de famílias de plantas daninhas por classe nos plantios de guaraná em cinco municípios do Estado do Amazonas - 2003

\begin{tabular}{|c|c|c|c|c|c|}
\hline \multirow{2}{*}{ Classe } & \multicolumn{5}{|c|}{ Número de família de plantas daninhas por classe e } \\
& Coor localidade \\
\cline { 2 - 6 } & Iranduba & Maués & $\begin{array}{c}\text { Presidente } \\
\text { Figueiredo }\end{array}$ & Urucará \\
\hline Dicotiledônea & 17 & 10 & 24 & 8 & 18 \\
\hline $\begin{array}{l}\text { Monocotiliedônea } \\
\text { Pteridófita }\end{array}$ & 2 & 1 & 2 & 2 & 2 \\
\hline Total & 2 & 1 & 1 & 1 & - \\
\hline
\end{tabular}

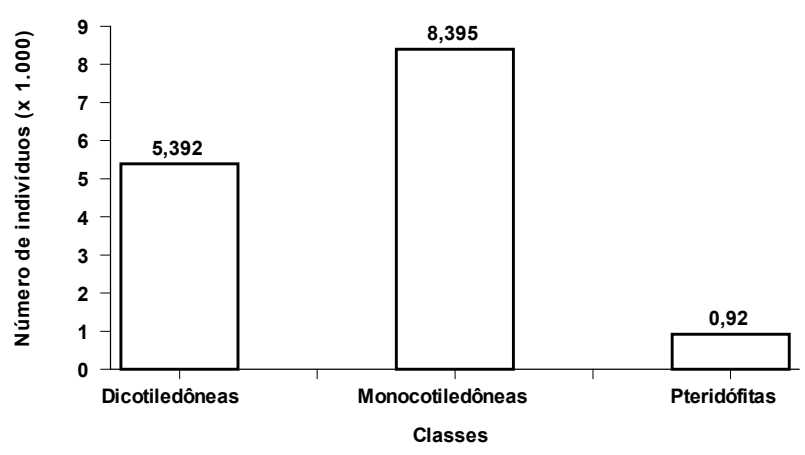

Figura 1 - Número de indivíduos por classe de planta nos cinco municípios estudados -2003. com 10 cada. Resultados semelhantes foram encontrados por Peixoto et al. (1982), os quais identificaram 27 familias, dentre estas Poaceae, que apresentou maior número de espécie em pastagem no município de Vassouras-RJ. No trabalho de Yanagizava \& Maimoni-Rodella (1999), as famílias Asteraceae e Poaceae também apresentaram maior número de espécies: 17 e 7 , respectivamente.

Poaceae apresentou o maior número de espécies, seguida das famílias Rubiaceae, Solanaceae, Clusiaceae e Fabaceae (Tabela 2). As outras famílias não ocorreram em todos os municípios, e mais da metade delas (55\%) estava representada por apenas uma espécie.

As seis espécies com maior número de indivíduos (Panicum pilosum, Scleria malaleuca, Panicum laxum, Spermacoce capitata, Selaginella asperula e Homolepis aturensis) continham mais da metade do total deles $(8.142$, equivalente a $55,36 \%$ ); os outros $44,64 \%$ ficaram distribuídos entre as outras 81 espécies restantes (Tabela 3).

A maioria das espécies apresentou freqüência muito baixa - inferior a $10 \%$ (Tabela 3). Destacaram-se como as espécies mais freqüentes: Panicum pilosum, Spermacoce capitata $(26,92 \%)$, Sabicea amazonensis (23,08\%), Scleria malaleuca (18,85\%), Panicum laxum (15,38\%) e Homolepis aturensis (14,23\%). Em relação ao número de plantas por área, do total de 87 espécies, 24 (28\%) apresentaram densidade entre 1 e 26 individuos, sendo o restante das espécies (63) com valor inferior a um indivíduo por metro quadrado. Esses resultados estão de acordo com os obtidos por Modesto Junior \& Mascarenhas (2001), que em pastagens no nordeste paraense registraram que a maior parte das espécies encontradas $(70 \%)$ apresentava baixa densidade de individuos e somente duas delas se destacaram com alta densidade.

A maior abundância foi apresentada por Stachytarpheta cayennensis, com 72,00 indivíduos por parcela; isso foi atribuído ao fato de esta espécie ter sido encontrada em apenas uma parcela em toda a área correspondente aos cinco municípios. Esse resultado sugere que a metodologia usada talvez não fosse a mais adequada para esse parâmetro.

Planta Daninha, Viçosa-MG, v. 22, n. 3, p. 351-358, 2004 
Tabela 2 - Número de espécies de plantas daninhas por família nos plantios de guaraná em cinco municípios do Estado do Amazonas - 2003

\begin{tabular}{|c|c|c|c|c|c|}
\hline \multirow{2}{*}{ Família } & \multicolumn{5}{|c|}{ Número de espécies de plantas daninhas por família e por localidade } \\
\hline & Coari & Iranduba & Maués & Presidente Figueiredo & Urucará \\
\hline Amaranthaceae & 1 & 0 & 1 & 0 & 0 \\
\hline Apocinaceae & 1 & 1 & 0 & 0 & 2 \\
\hline Asteraceae & 2 & 3 & 7 & 3 & 0 \\
\hline Bignoniaceae & 2 & 0 & 2 & 1 & 3 \\
\hline Boraginaceae & 0 & 0 & 1 & 0 & 0 \\
\hline Caesalpiniaceae & 0 & 0 & 2 & 0 & 0 \\
\hline Clusiaceae & 2 & 1 & 1 & 1 & 1 \\
\hline Cyperaceae & 2 & 0 & 2 & 1 & 3 \\
\hline Davalliaceae & 1 & 0 & 0 & 0 & 0 \\
\hline Dennstaediaceae & 0 & 1 & 0 & 0 & 0 \\
\hline Dilleniaceae & 1 & 0 & 2 & 1 & 1 \\
\hline Euphorbiaceae & 2 & 0 & 2 & 0 & 1 \\
\hline Fabaceae & 1 & 1 & 1 & 1 & 1 \\
\hline Leguminoseae & 0 & 0 & 0 & 1 & 1 \\
\hline Melastomataceae & 2 & 1 & 1 & 0 & 0 \\
\hline Menispermaceae & 0 & 1 & 1 & 0 & 0 \\
\hline Passifloraceae & 1 & 0 & 1 & 0 & 1 \\
\hline Piperaceae & 1 & 0 & 2 & 0 & 0 \\
\hline Poaceae & 4 & 4 & 8 & 5 & 6 \\
\hline Pteridaceae & 2 & 0 & 1 & 1 & 0 \\
\hline Rubiaceae & 2 & 2 & 2 & 2 & 2 \\
\hline Rutaceae & 0 & 0 & 1 & 0 & 1 \\
\hline Solanaceae & 2 & 2 & 1 & 2 & 1 \\
\hline Theophrastaceae & 0 & 1 & 1 & 0 & 0 \\
\hline Verbenaceae & 0 & 1 & 1 & 0 & 0 \\
\hline
\end{tabular}

Tabela 3 - Classes, famílias, espécies, número de indivíduos e número de parcelas de plantas daninhas em plantios de guaraná e seus parâmetros, avaliados de forma conjunta para cinco municípios do Estado do Amazonas - 2003

\begin{tabular}{|c|c|c|c|c|c|c|c|c|c|c|c|}
\hline Classe & Família & Espécie & N.I. ${ }^{1 /}$ & N.P. ${ }^{2 /}$ & Fre $\% 0^{\frac{3}{6}}$ & $\operatorname{Den}^{4 /}$ & $\mathrm{Abu}^{5 /}$ & Frer $^{6 /}$ & $\operatorname{Denr}^{7 /}$ & Abur $^{8 /}$ & $\mathrm{IVI}^{9 /}$ \\
\hline $\mathrm{M}$ & Poaceae & Panicum pilosum $\mathrm{Sw}$. & 2342 & 70 & 26,92 & 25,02 & 33,46 & 6,97 & 15,92 & 4,46 & 27,36 \\
\hline M & Cyperaceae & Scleria melaleuca Schlect. \& Cham. & 1506 & 49 & 18,85 & 16,09 & 30,73 & 4,88 & 10,24 & 4,10 & 19,22 \\
\hline $\mathrm{M}$ & Poaceae & Panicum laxum Sw. & 1439 & 40 & 15,38 & 15,37 & 35,98 & 3,98 & 9,78 & 4,80 & 18,56 \\
\hline $\mathrm{D}$ & Rubiaceae & Spermacoce capitata (RUIZ \& PAV.) DC. & 1057 & 70 & 26,92 & 11,29 & 15,10 & 6,97 & 7,19 & 2,01 & 16,17 \\
\hline$P$ & Pteridaceae & Selaginella asperula Spring & 903 & 30 & 11,54 & 9,65 & 30,10 & 2,99 & 6,14 & 4,01 & 13,14 \\
\hline M & Poaceae & Homolepis aturensis (H.B.K.) Chase & 895 & 37 & 14,23 & 9,56 & 24,19 & 3,68 & 6,09 & 3,22 & 12,99 \\
\hline D & Euphorbiaceae & Chamaesyce hirta Millsp. & 682 & 20 & 7,69 & 7,29 & 34,10 & 1,99 & 4,64 & 4,55 & 11,17 \\
\hline $\mathrm{D}$ & Rubiaceae & Sabicea amazonensis Wernh. & 500 & 60 & 23,08 & 5,34 & 8,33 & 5,98 & 3,40 & 1,11 & 10,49 \\
\hline $\mathrm{D}$ & Verbenaceae & Stachytarpheta cayennensis (Rich.) Vahl & 72 & 1 & 0,38 & 0,77 & 72,00 & 0,10 & 0,49 & 9,60 & 10,19 \\
\hline D & Asteraceae & Unxia canphorata L. f. & 525 & 32 & 12,31 & 5,61 & 16,41 & 3,19 & 3,57 & 2,19 & 8,94 \\
\hline $\mathrm{M}$ & Poaceae & Homolepis sp. & 497 & 20 & - 7,69 & 5,31 & 24,85 & 1,99 & 3,38 & 3,31 & 8,68 \\
\hline M & Poaceae & Paspalum conjugatum Berg & 486 & 34 & 13,08 & 5,19 & 14,29 & 3,39 & 3,30 & 1,90 & 8,60 \\
\hline M & Cyperaceae & Rhynchospora nervosa (Vahl) Boeckeler & 369 & 29 & 11,15 & 3,94 & 12,72 & 2,89 & 2,51 & 1,70 & 7,09 \\
\hline M & Poaceae & Paspalum virgatum $\mathrm{L}$. & 291 & 17 & 6,54 & 3,11 & 17,12 & 1,69 & 1,98 & 2,28 & 5,95 \\
\hline M & Poaceae & Brachiaria decunbens Stapf & 229 & 16 & 6,15 & 2,45 & 14,31 & 1,59 & 1,56 & 1,91 & 5,06 \\
\hline $\mathrm{D}$ & Scrophulariaceae & Scoparia dulcis $\mathrm{L}$. & 145 & 21 & 8,08 & 1,55 & 6,90 & 2,09 & 0,99 & 0,92 & 4,00 \\
\hline $\mathrm{D}$ & Melastomataceae & Clidemia rubra (Aubl.) Mart. & 122 & 24 & 9,23 & 1,30 & 5,08 & 2,39 & 0,83 & 0,68 & 3,90 \\
\hline D & Amaranthaceae & Cyathula prostata Blume. & 17 & 4 & 1,54 & 0,18 & 4,25 & 0,40 & 0,12 & 0,57 & 1,08 \\
\hline D & Verbenaceae & Lantana câmara L. & 12 & 6 & 2,31 & 0,13 & 2,00 & 0,60 & 0,08 & 0,27 & 0,95 \\
\hline $\mathrm{D}$ & Passifloraceae & Passiflora coccinea Aubl. & 9 & 4 & 1,54 & 0,10 & 2,25 & 0,40 & 0,06 & 0,30 & 0,76 \\
\hline $\mathrm{D}$ & Flarcourtiaceae & Casearia javitensis H.B.K & 4 & 1 & 0,38 & 0,04 & 4,00 & 0,10 & 0,03 & 0,53 & 0,66 \\
\hline $\mathrm{P}$ & Dennstaediaceae & Pteridium aquilinum (L.) Kuhn & 6 & 3 & 1,15 & 0,06 & 2,00 & 0,30 & 0,04 & 0,27 & 0,61 \\
\hline D & Dilleniaceae & Doliopcarpus magnificus Sluim. & 4 & 2 & 0,77 & 0,04 & 2,00 & 0,20 & 0,03 & 0,27 & 0,49 \\
\hline D'm & Asteraceae & Bidens pilosa L. & 1 & 1 & 0,38 & 0,01 & 1,00 & 0,10 & 0,01 & 0,13 & 0,24 \\
\hline
\end{tabular}

${ }^{1 /}$ número de indivíduos; ${ }^{2 /}$ número de parcelas; ${ }^{3 /}$ freqüência; ${ }^{4 /}$ densidade; ${ }^{5 /}$ abundância; ${ }^{6 /}$ freqüência relativa; ${ }^{7 /}$ densidade relativa; ${ }^{8 /}$ abundância relativa; ${ }^{9 /}$ índice de valor de importância. 
A menor abundância $(1,00)$ ficou para as espécies representadas por um único individuo (Tabela 3).

Panicum pilosum destacou-se como a espécie mais importante, com IVI igual a 27,36, seguida de Scleria malaleuca $(19,22)$, Panicum laxum $(18,56)$, Spermacoce capitata $(16,17)$, Selaginella asperula $(13,14)$ e Homolepis aturensis $(12,99)$. Paspalum conjugatum, Cyathula prostata e Bidens pilosa, com IVI de $8,60,1,08$ e 0,24, respectivamente, apesar de não estarem entre as mais importantes exceto Paspalum conjugatum, que apareceu em $12^{\circ}$ lugar (Tabela 3) - são citadas por Freire et al. (1988) como entre as dez principais plantas daninhas que ocorrem em guaranazais da região sudeste da Bahia.

Os cinco municípios apresentaram variação quanto à composição florística, e a maior diversidade de espécies foi encontrada em Maués (47), seguido de Coari (35), Urucará (31), Iranduba e Presidente Figueiredo, com 19 espécies cada (Tabela 4 ).

Quanto ao número de indivíduos, Coari destacou-se, com 4.706, vindo em seguida Maués (3.824), Urucará, (3.121), Presidente Figueiredo (2.723) e Iranduba (296) (Tabela 4). Entretanto, esta tabela contém apenas as espécies de maior IVI para cada município; as demais estão relacionadas no trabalho de Albertino (2003).

As Dicotiledôneas superaram as Monocotiledôneas e Pteridófitas em número de indivíduos em dois municípios: Maués, com 591 (62\%), e Iranduba, onde ocorreram 168 indivíduos, que representavam 57\% do total de individuos das três classes juntas (Figura 2).

As Monocotiledôneas prevaleceram em Presidente Figueiredo, Coari e Urucará. Destes três municipios, Presidente Figueiredo destacou-se em primeiro lugar, com $70 \%$ do total de individuos pertencentes a esta classe (954); em Coari ela representou 64\% dos indivíduos (1004), e em Urucará, 61\% (632) (Figura 2).

As Pteridófitas ocorreram de forma inexpressiva em todos os municípios, e em Presidente Figueiredo apresentou maior ocorrência: 184 indivíduos (14\%) (Figura 2).
Poaceae foi a família que apresentou maior número de espécies e de indivíduos em todos os municipios, exceto em Coari, onde era a primeira em número de espécies e a segunda em número de indivíduos (1.492), superada pela Cyperaceae (1.517). Neste municipio, estas famílias representaram $64 \%$ do total de indivíduos registrados para as 22 existentes. Em Presidente Figueiredo, Poaceae se destacou com 1.852 indivíduos (68\%); em Urucará, com 1.725 (55\%); em Iranduba, com 122 (41\%); e em Maués, com 1.161 (30\%) (Tabela 4).

Em Maués foi registrada, para Poaceae, a maior ocorrência de espécies (oito), seguida de Urucará com seis e Presidente Figueiredo com cinco. A menor ocorrência foi de quatro espécies, em Coari e Iranduba (Tabela 2).

As espécies que sobressaíram quanto ao número de indivíduos foram: Scleria malaleuca (1.384), em Coari; Emilia sonchifolia (56), em Iranduba; Chamaesyce hirta (683), em Maués; Panicum pilosum (1.058), em Presidente Figueiredo; e Panicum laxum (1.363), em Urucará (Tabela 4).

Homolepis aturensis, Paspalum conjugatum e Spermacoce capitata foram espécies que ocorreram nos cinco municípios (Tabela 4).

Quanto à freqüência, Panicum pilosum se destacou em Coari, onde estava distribuída em $80 \%$ da área, e em Presidente Figueiredo, como a segunda espécie mais freqüente $(42,50 \%)$. Sabicea amazonensis foi a mais freqüente em Maués $(27,5 \%)$ e a segunda em Coari (62\%); já em Urucará ela apareceu com a menor freqüência $(1,67 \%)$. Spermacoce capitata foi a mais freqüente em Urucará $(63,33 \%)$ e a terceira em Presidente Figueiredo (25\%), porém uma das menos frequentes em Iranduba (5\%). Homolepis aturensis obteve maior freqüência em Presidente Figueiredo (52,50\% da área) e, em Iranduba, era a quarta espécie mais frequente $(20 \%)$ - a maior freqüência encontrada neste municipio foi para Homolepis sp., em 50\% da área (Tabela 4).

Panicum pilosum foi a mais densa em Presidente Figueiredo, com 73,47 indivíduos por $\mathrm{m}^{2}$ - sobressaiu também em Coari, como a segunda mais densa $(58,01)$; Chamaesyce hirta $(23,72)$ foi a mais densa de Maués; Emilia sonchifolia $(7,78)$, em Iranduba; Scleria malaleuca (64,07), em Coari; e Panicum laxum,

Planta Daninha, Viçosa-MG, v. 22, n. 3, p. 351-358, 2004 
Tabela 4 - Classes, famílias, espécies, número de indivíduos e número de parcelas de plantas daninhas em plantios de guaraná e seus parâmetros, avaliados de forma conjunta para cada município estudado do Estado do Amazonas - 2003

\begin{tabular}{|c|c|c|c|c|c|c|c|c|c|c|c|c|}
\hline Município & Classe & Família & Espécie & N.I. ${ }^{1 /}$ & N.P. ${ }^{\prime \prime}$ & $\mathrm{Fre} \%{ }^{3 /-}$ & $\operatorname{Den}^{4 /}$ & $\mathrm{Abu}^{\frac{5 /}{2}}$ & $\mathrm{Frer}^{6 /}$ & Denr $^{7 /}$ & Abur $^{8 /}$ & $\mathrm{IVI}^{\mathrm{g}^{9}}$ \\
\hline \multirow{12}{*}{ Coari } & $\mathrm{M}$ & Cyperaceae & Scleria malaleuca Schlect. \& Cham. & 1.384 & 29 & 48,00 & 64,07 & 47,72 & 8,92 & 29,43 & 16,38 & 54,73 \\
\hline & $\mathrm{M}$ & Poaceae & Panicum pilosum Sw. & 1.253 & 48 & 80,00 & 58,01 & 26,10 & 14,77 & 26,64 & 8,96 & 50,37 \\
\hline & $\mathrm{P}$ & Pteridaceae & Selaginella asperula Spring. & 503 & 20 & 33,00 & 23,29 & 25,15 & 6,15 & 10,70 & 8,63 & 25,48 \\
\hline & $\mathrm{D}$ & Rubiaceae & Sabicea amazonensis Wernh. & 221 & 37 & 62,00 & 10,23 & 5,97 & 11,38 & 4,70 & 2,05 & 18,13 \\
\hline & M & Poaceae & Paspalum conjugatum Berg. & 212 & 12 & 20,00 & 9,81 & 17,67 & 3,69 & 4,51 & 6,06 & 14,26 \\
\hline & $\mathrm{D}$ & Euphorbiaceae & Phyllanthus niruri $\mathrm{L}$. & 186 & 16 & 27,00 & 8,61 & 11,63 & 4,92 & 3,95 & 3,99 & 12,87 \\
\hline & D & Fabaceae & Pueraria phaseoloides (Roxb.) Benth. & 76 & 3 & 5,00 & 3,52 & 25,33 & 0,92 & 1,62 & 8,69 & 11,23 \\
\hline & $\mathrm{D}$ & Melastomataceae & Clidemia rubra (Aubl.) Mart. & 106 & 15 & 25,00 & 4,91 & 7,07 & 4,62 & 2,25 & 2,43 & 9,29 \\
\hline & D & Rubiaceae & Spermacoce capitata (RUIZ \& PAV.) DC. & 61 & 10 & 17,00 & 2,82 & 6,10 & 3,08 & 1,30 & 2,09 & 6,47 \\
\hline & M & Poaceae & Homolepis aturensis (H.B.K.) Chase & 15 & 1 & 2,00 & 0,69 & 15,00 & 0,31 & 0,32 & 5,15 & 5,77 \\
\hline & D & Amaranthaceae & Cyathula prostata Blume. & 4 & 1 & 2,00 & 0,19 & 4,00 & 0,31 & 0,09 & 1,37 & 1,77 \\
\hline & M & Cyperaceae & Cyperus luzulae Rotz. & 4 & 2 & 3,00 & 0,19 & 2,00 & 0,62 & 0,09 & 0,69 & 1,39 \\
\hline \multirow{13}{*}{ Iranduba } & $\mathrm{M}$ & Poaceae & Panicum laxum $\mathrm{Sw}$. & 48 & 1 & 5,00 & 6,67 & 48,00 & 1,64 & 16,44 & 47,65 & 65,73 \\
\hline & $\mathrm{D}$ & Asteraceae & Emilia sonchifolia (L.) DC. & 56 & 7 & 35,00 & 7,78 & 8,00 & 11,48 & 19,18 & 7,94 & 38,59 \\
\hline & $\mathrm{M}$ & Poaceae & Homolepis sp. & 50 & 10 & 50,00 & 6,94 & 5,00 & 16,39 & 17,12 & 4,96 & 38,48 \\
\hline & $\mathrm{D}$ & Cluseaceae & Vismia cayennensis (jacq.) Pers. & 46 & 7 & 35,00 & 6,39 & 6,57 & 11,48 & 15,75 & 6,52 & 33,75 \\
\hline & $\mathrm{D}$ & Asteraceae & Eupatorium sp. & 21 & 7 & 35,00 & 2,92 & 3,00 & 11,48 & 7,19 & 2,98 & 21,65 \\
\hline & $M$ & Poaceae & Homolepis aturensis (H.B.K.) Chase & 22 & 4 & 20,00 & 3,06 & 5,50 & 6,56 & 7,53 & 5,46 & 19,55 \\
\hline & $\mathrm{D}$ & Verbenaceae & Lantana camara $\mathrm{L}$. & 12 & 6 & 30,00 & 1,67 & 2,00 & 9,84 & 4,11 & 1,99 & 15,93 \\
\hline & $\mathrm{D}$ & Solanaceae & Solanum rugosum Dunal. & 5 & 3 & 15,00 & 0,69 & 1,67 & 4,92 & 1,71 & 1,65 & 8,28 \\
\hline & $\mathrm{D}$ & Melastomataceae & Clidemia rubra (Aubl.) Mart. & 4 & 1 & 5,00 & 0,56 & 4,00 & 1,64 & 1,37 & 3,97 & 6,98 \\
\hline & $\mathrm{D}$ & Solanaceae & Solanum subinerme Jacq. & 3 & 2 & 10,00 & 0,42 & 1,50 & 3,28 & 1,03 & 1,49 & 5,80 \\
\hline & M & Poaceae & Paspalum conjugatum Berg. & 2 & 2 & 10,00 & 0,28 & 1,00 & 3,28 & 0,68 & 0,99 & 4,96 \\
\hline & D & Menispermaceae & Cissanpelos sp. & 1 & 1 & 5,00 & 0,14 & 1,00 & 1,64 & 0,34 & 0,99 & 2,97 \\
\hline & D & Rubiaceae & Spermacoce capitata (RUIZ \& PAV.) DC. & 1 & 1 & 5,00 & 0,14 & 1,00 & 1,64 & 0,34 & 0,99 & 2,97 \\
\hline \multirow{12}{*}{ Maués } & $\mathrm{D}$ & Euphorbiaceae & Chamaesyce hirta Millsp. & 683 & 21 & 26,25 & 23,72 & 32,52 & 6,55 & 17,86 & 7,37 & 31,77 \\
\hline & $\mathrm{M}$ & Poaceae & Homolepis sp. & 447 & 10 & 12,50 & 15,52 & 44,70 & 3,12 & 11,69 & 10,12 & 24,93 \\
\hline & $\mathrm{D}$ & Asteraceae & Unxia canphorata L. f. & 444 & 14 & 17,50 & 15,42 & 31,71 & 4,36 & 11,61 & 7,18 & 23,16 \\
\hline & D & Verbenaceae & Stachytarpheta cayennensis (Rich.) Vahl & 74 & 1 & 1,25 & 2,57 & 74,00 & 0,31 & 1,94 & 16,76 & 19,01 \\
\hline & $\mathrm{D}$ & Rubiaceae & Sabicea amazonensis Wernh. & 258 & 22 & 27,50 & 8,96 & 11,73 & 6,86 & 6,75 & 2,66 & 16,26 \\
\hline & M & Cyperaceae & Scleria malaleuca Schlect. \& Cham. & 114 & 19 & 23,75 & 3,96 & 6,00 & 5,92 & 2,98 & 1,36 & 10,26 \\
\hline & $\mathrm{M}$ & Poaceae & Paspalum conjugatum Berg. & 61 & 11 & 13,75 & 2,12 & 5,55 & 3,43 & 1,60 & 1,26 & 6,28 \\
\hline & $\mathrm{D}$ & Rubiaceae & Spermacoce capitata (RUIZ \& PAV.) DC. & 50 & 11 & 13,75 & 1,74 & 4,55 & 3,43 & 1,31 & 1,03 & 5,77 \\
\hline & $\mathrm{M}$ & Poaceae & Homolepis aturensis (H.B.K.) Chase & 52 & 8 & 10,00 & 1,81 & 6,50 & 2,49 & 1,36 & 1,47 & 5,33 \\
\hline & $\mathrm{P}$ & Pteridaceae & Selaginella asperula Spring. & 32 & 3 & 3,75 & 1,11 & 10,67 & 0,94 & 0,84 & 2,42 & 4,19 \\
\hline & $\mathrm{D}$ & Amaranthaceae & Cyathula prostata Blume. & 30 & 7 & 8,75 & 1,04 & 4,29 & 2,18 & 0,78 & 0,97 & 3,94 \\
\hline & $\mathrm{M}$ & Poaceae & Panicum laxum $\mathrm{Sw}$. & 28 & 5 & 6,25 & 0,97 & 5,60 & 1,56 & 0,73 & 1,27 & 3,56 \\
\hline \multirow{12}{*}{$\begin{array}{l}\text { Presidente } \\
\text { Figueiredo }\end{array}$} & $\mathrm{M}$ & Poaceae & Panicum pilosum $\mathrm{Sw}$. & 1.058 & 17 & 42,50 & 73,47 & 62,24 & 13,60 & 38,85 & 23,32 & 75,77 \\
\hline & $\mathrm{M}$ & Poaceae & Homolepis aturensis (H.B.K.) Chase & 686 & 21 & 52,50 & 47,64 & 32,67 & 16,80 & 25,19 & 12,24 & 54,23 \\
\hline & P & Pteridaceae & Selaginella asperula Spring. & 368 & 7 & 17,50 & 25,56 & 52,57 & 5,60 & 13,51 & 19,70 & 38,81 \\
\hline & M & Poaceae & Paspalum conjugatum Berg. & 90 & 6 & 15,00 & 6,25 & 15,00 & 4,80 & 3,31 & 5,62 & 13,73 \\
\hline & D & Rubiaceae & Spermacoce capitata (RUIZ \& PAV.) DC. & 75 & 10 & 25,00 & 5,21 & 7,50 & 8,00 & 2,75 & 2,81 & 13,56 \\
\hline & $\mathrm{M}$ & Cyperaceae & Cyperus luzulae Rotz. & 56 & 4 & 10,00 & 3,89 & 14,00 & 3,20 & 2,06 & 5,25 & 10,50 \\
\hline & $\mathrm{D}$ & Leguminoseae & Zornia latifolia $\mathrm{Sm}$. & 34 & 2 & 5,00 & 2,36 & 17,00 & 1,60 & 1,25 & 6,37 & 9,22 \\
\hline & M & Poaceae & Paspalum virgatum $\mathrm{L}$. & 17 & 1 & 2,50 & 1,18 & 17,00 & 0,80 & 0,62 & 6,37 & 7,79 \\
\hline & $\mathrm{D}$ & Solanaceae & Solanum subinerme Jacq. & 10 & 5 & 12,50 & 0,69 & 2,00 & 4,00 & 0,37 & 0,75 & 5,12 \\
\hline & $\mathrm{D}$ & Bignoniaceae & Martinella obovata (H.B.K.) Sandwith. & 8 & 4 & 10,00 & 0,56 & 2,00 & 3,20 & 0,29 & 0,75 & 4,24 \\
\hline & D & Cluseaceae & Vismia cayennensis (jacq.) Pers. & 5 & 3 & 7,50 & 0,35 & 1,67 & 2,40 & 0,18 & 0,62 & 3,21 \\
\hline & D & Solanaceae & Solanum rugosum Dunal. & 3 & 2 & 5,00 & 0,21 & 1,50 & 1,60 & 0,11 & 0,56 & 2,27 \\
\hline \multirow{8}{*}{ Urucará } & $\mathrm{M}$ & Poaceae & Panicum laxum $\mathrm{Sw}$. & 1.363 & 34 & 56,67 & 63,10 & 40,09 & 18,89 & 43,67 & 11,82 & 74,38 \\
\hline & D & Rubiaceae & Spermacoce capitata (RUIZ \& PAV.) DC. & 870 & 38 & 63,33 & 40,28 & 22,89 & 21,11 & 27,88 & 6,75 & 55,74 \\
\hline & $\mathrm{M}$ & Poaceae & Paspalum conjugatum Berg. & 121 & 3 & 5,00 & 5,60 & 40,33 & 1,67 & 3,88 & 11,89 & 17,43 \\
\hline & $\mathrm{M}$ & Poaceae & Homolepis aturensis (H.B.K.) Chase & 120 & 3 & 5,00 & 5,56 & 40,00 & 1,67 & 3,84 & 11,79 & 17,30 \\
\hline & D & Rubiaceae & Sabicea amazonensis Wernh. & 21 & 1 & 1,67 & 0,97 & 21,00 & 0,56 & 0,67 & 6,19 & 7,42 \\
\hline & M & Poaceae & Panicum pilosum Sw. & 31 & 5 & 8,33 & 1,44 & 6,20 & 2,78 & 0,99 & 1,83 & 5,60 \\
\hline & $\mathrm{M}$ & Cyperaceae & Scleria malaleuca Schlect. \& Cham. & 8 & 1 & 1,67 & 0,37 & 8,00 & 0,56 & 0,26 & 2,36 & 3,17 \\
\hline & D & Passifloraceae & Passiflora coccinea Aubi & 2 & 1 & 1,67 & 0,09 & 2,00 & 0,56 & 0,06 & 0,59 & 1,21 \\
\hline
\end{tabular}

${ }^{1 /}$ número de indivíduos; ${ }^{2 /}$ número de parcelas; ${ }^{3 /}$ freqüência; ${ }^{4 /}$ densidade; ${ }^{5 /}$ abundância; ${ }^{6 /}$ freqüência relativa; ${ }^{7 /}$ densidade relativa;

8/ abundância relativa; ${ }^{2 /}$ índice de valor de importância. 


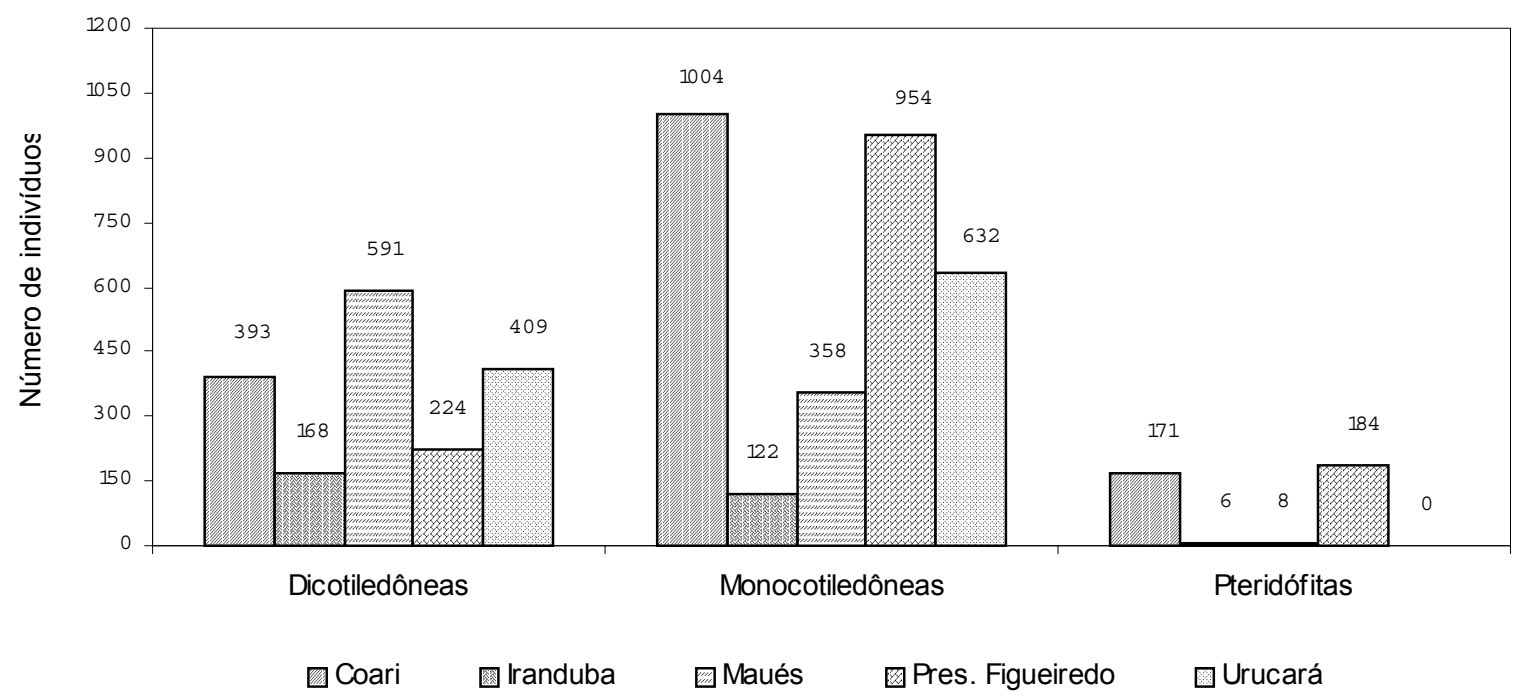

Figura 2 - Número de indivíduos por classe em cada município estudado do Estado do Amazonas (n=20).

em Urucará, com 63,10 indivíduos por $\mathrm{m}^{2}$ (Tabela 4).

O parâmetro abundância revelou como mais abundantes as espécies Stachytarpheta cayennensis, em Maués, com 74 indivíduos por parcela; Panicum pilosum (62,24), em Presidente Figueiredo e em Coari $(26,10)$; Panicum laxum (48,00 e 40,09), em Iranduba e Urucará, respectivamente; Scleria malaleuca $(47,72)$, em Coari; e Rhynchospora nervosa $(43,50)$, em Urucará (Tabela 4). No entanto, é importante observar que a maior abundância de Stachytarpheta cayennensis e Rhynchospora nervosa nesses municipios se deve à sua ocorrência em poucas parcelas; já Panicum laxum, em Urucará, e Panicum pilosum, em Coari e Presidente Figueiredo, podem admitir métodos próprios para seu controle, pois são espécies que sobressaem tanto em número de individuos quanto em número de parcelas ocupadas.

Panicum pilosum foi a espécie mais importante no municipio de Presidente Figueiredo, com o índice de valor de importância (IVI) igual a 75,77; também apareceu em Coari, como a segunda mais importante $(50,37)$. Panicum laxum sobressaiu em Urucará e Iranduba, com IVI igual a 74,38 e 65,73, respectivamente. Scleria malaleuca destacou-se em Coari, com IVI de 54,73. Em Maués o destaque foi para Chamaesyce hirta, cujo IVI atingiu 31,77 (Tabela 4). A ocorrência de mesma espécie em dois ou mais municípios indica a dispersão desta na área amostrada.
O teste de Kruskall-Wallis mostrou diferença por número de indivíduos entre municípios para Clidemia rubra $(\mathrm{p}=0,031)$, Panicum laxum $(\mathrm{p}=0,048)$, Panicum pilosum $(\mathrm{p}=0,001)$, Paspalum conjugatum $(\mathrm{p}=0,001)$, Scleria malaleuca $(\mathrm{p}=0,001)$, Spermacoce capitata $(\mathrm{p}=0,004)$ e Unxia canphorata $(\mathrm{p}=0,017)$. Para as demais espécies não foi encontrada diferença entre os municipios.

Alguns municipios mostraram maiores semelhanças em termos de espécies comuns e poucas espécies comuns com outros (Tabela 5). No entanto, o índice de similaridade é baixo entre eles, sendo todos menores que $50 \%$, o que pode ser explicado pelo fato de que muitas espécies apresentaram-se exclusivas em cada município avaliado.

Os maiores indices de similaridade foram entre os municipios de Iranduba e Presidente Figueiredo $(45,00 \%)$ e entre Coari e Urucará $(44,12 \%)$. Os menores indices foram entre Iranduba e Urucará $(26,92 \%)$ e Iranduba e Coari $(21,43 \%)$.

Carvalho \& Pitelli (1992) afirmaram que os índices de similaridade não estão relacionados aos solos ou à distância entre áreas, mas podem estar ligados às formas de manejo empregadas nessas áreas. Os resultados encontrados tendem a ser considerados como concordantes com os desses autores, uma vez que nos municípios de Iranduba e Presidente Figueiredo a forma de manejo da cultura do guaraná é

Planta Daninha, Viçosa-MG, v. 22, n. 3, p. 351-358, 2004 
Tabela 5 - Índice de similaridade (\%) de espécies de plantas daninhas nos plantios de guaraná em cinco municípios do Estado do Amazonas, 2003

\begin{tabular}{|l|c|c|c|c|}
\hline \multicolumn{1}{|c|}{ Município } & Iranduba & Maués & $\begin{array}{c}\text { Presidente } \\
\text { Figueiredo }\end{array}$ & Urucará \\
\hline Coari & 21,43 & 39,53 & 28,57 & 44,12 \\
\hline Iranduba & - & 28,57 & 45,00 & 26,92 \\
\hline Maués & - & - & 28,57 & 36,59 \\
\hline Presidente Figueiredo & - & - & - & 30,77 \\
\hline
\end{tabular}

semelhante, com o mínimo de perturbação das populações de plantas daninhas.

A maioria das espécies de plantas daninhas não ocorreu em todos os municípios, sendo a maior diversidade encontrada em Maués.

A maior ocorrência de espécies foi verificada na classe das Dicotiledôneas e nas famílias Poaceae e Asteraceae, enquanto Panicum pilosum, Scleria malaleuca, Panicum laxum, Spermacoce capitata, Selaginella asperula e Homolepis aturensis foram consideradas as mais importantes. O maior número de indivíduos ocorreu no município de Coari e o menor em Iranduba; já o maior índice de similaridade entre as espécies foi observado em Presidente Figueiredo e Iranduba, e o menor, entre Coari e Iranduba.

\section{LITERATURA CITADA}

ALBERTINO, S. M. F. Composição florística de plantas daninhas em plantios de guaraná (Paullinia cupana var. Sorbilis), no estado do amazonas. 2003. $45 \mathrm{f}$. Dissertação (Mestrado em Sistemas Agroflorestais) Universidade Federal do Amazonas, Manaus, 2003.

ALCANTARA, E. M.; CARVALHO, D. A. Plantas daninhas em mandiocais (Manihot sculenta Crantz) na Região Mineradora de Diamantina (Alto Jequitinhonha) Minas Gerais. Planta Daninha, v. 6, n. 2, p. 138-143, 1983.

BRANDÃO, M.; BRANDÃO, H.; LACA-BUENDIA, J. P. A mata ciliar do Rio Sapucaí-MG: fitossociologia. Daphne, v. 8, n. 4, p. 36-48, 1998.

BRANDÃO, M.; GAVILANES, M. L.; DEL LACABUENDIA, J. P. C. Levantamento e identificação de plantas daninhas na cultura do feijão (Phaseolus vulgaris L.) no Estado de Minas Gerais. In: CONGRESSO BRASILEIRO DE HERBICIDAS E PLANTAS DANINHAS, 16., 1986, Campo Grande. Resumos... Campo Grande: 1986. p. 19.
CAMPELO, C. R. Plantas daninhas da cultura da cana-deaçúcar (Saccharum sp.) no Estado de Alagoas. In: CONGRESSO BRASILEIRO DE HERBICIDAS E PLANTAS DANINHAS, 17., 1988, Piracicaba, SP. Resumos... Piracicaba: 1988. p. 25-26.

CARVALHO, S. L.; PITELLI, R. A. Comportamento e análise fitossociológica das principais espécies de plantas daninhas de pastagens da região de Selvia, MS. Planta Daninha, v. 10, n. 1-2, p. 25-32, 1992.

DOMBROSWSKI, L. T. D. Gramíneas no Paraná. Londrina: IAPAR, 1989. p. 110-116 (Boletim Técnico, 28)

FREIRE, A. S.; PEREIRA, R.C.; SACRAMENTO, C. K. Efeito de herbicidas em plântulas de guaraná (Paullinia cupana var. sorbilis (Mart) Ducke) e sobre as principais plantas daninhas ocorrentes na cultura. Theobroma, v. 18, n. 1, p. $67-81,1988$.

LEITÃO, H. F.; ARANHA, C.; BACCHI, O. Plantas invasoras de culturas no Estado de São Paulo. São Paulo: HUCITEC, v. 1. 1972. p. 1-291.

MODESTO JUNIOR, M. S.; MASCARENHAS, R. E. B. Levantamento da infestação de plantas daninhas: associadas a uma pastagem cultivada de baixa produtividade no nordeste paraense. Planta Daninha, v. 19, n. 1, p. 11-21, 2001.

PEIXOTO, A. L.; CARVAlHO, S. M.; ROSA, M. M. T. Análise botânica de um campo de pastagem no Estado do Rio de Janeiro. Planta Daninha, v. 5, n. 2, p. 1-7, 1982.

PEREIRA, E. S.; VELINI, E. D.; CARVALHO, L. R. Avaliações qualitativas e quantitativas de plantas daninhas na cultura da soja submetida aos sistemas de plantio direto e convencional. Planta Daninha, v. 18, n. 2, p. 207-216, 2000 .

SORENSEN, T. A method of stablishing groups of equal amplitude in plant society of species content. In: ODUM, E. P. Ecologia. México: Interamericana, 1972. 640 p.

SOUZA, G. F. Manejo do solo e seu efeito no crescimento e produção do cupuaçuzeiro (Theobroma grandiflorum (Willdenow ex Spreng) Schumann), na camposição florística e biomassa da parte aérea de plantas invasoras em sistemas agroflorestais no município de Presidente Figueiredo, Estado do Amazonas. 2000. 152 f. Tese (Mestrado em Botânica) Instituto Nacional de Pesquisas da Amazônia/ Fundação Universidade do Amazonas, Manaus, 2000.

SOUZA, L. S. A.; SILVA, J. F.; SOUZA M. D. B. Composição florística de plantas daninhas em agrossistemas de cupuaçuzeiro (Theobroma grandiflorum) e pupunheira (Bactris gasipaes). Planta Daninha, v. 21, n. 2, p. 249255, 2003.

YANAGIZAWA, Y. A. N. P.; MAIMONI-RODELLA, R. C. S. Composição florística e estrutura da comunidade de plantas do estrato herbáceo em áreas de cultivo de árvores frutíferas. Planta Daninha, v. 17, n. 3, p. 459-468, 1999. 